\section{Raloxifeno e Osteoporose: Revisão de Um Novo Modulador Seletivo do Receptor de Estrógeno}

revisão

\author{
RESUMO
}

Raloxifeno é um modulador seletivo do receptor de estrógeno de segunda geração com ação agonista no osso e sistema cardiovascular e ação antagonista na mama e útero. Sua seletividade tecidual ocorre devido a diversos mecanismos como diferentes receptores de estrógenos, distribuição diferencial destes receptores, diferentes co-fatores protéicos transcricionais e diferente conformação do receptor após ligação de raloxifeno. No osso, raloxifeno aumenta a massa óssea na coluna, fêmur, corpo inteiro. é eficaz em prevenir osteoporose em mulheres na pósmenopausa e reduz a incidência de fraturas vertebrais em $50 \%$ em mutheres com osteoporose. No sistema cardiovascular, raloxifeno reduz 0 colesterol total, LDL-colesterol, fibrinogênio e lipoproteína (a), não tendo efeito nos triglicérides e HDL-colesterol total, porém aumenta a subfração $\mathrm{HDL}-\mathrm{C}_{2}$. Raloxifeno tem atividade antiproliferativa na mama, näo induz mastalgia e uma redução na incidência de novos casos de câncer de mama tem sido demonstrada em mulheres em uso de raloxifeno em grandes estudos clínicos para osteoporose. No útero, raloxifeno não estimula o endométrio e não aumenta a incidência de sangramento vaginal ou carcinoma endometrial. $O$ evento adverso mais comum com raloxifeno são ondas de calor e o mais sério é o tromboembolismo venoso com incidência semelhante à terapia de reposição hormonal. Raloxifeno é uma alternativa para o tratamento e prevenção de osteoporose em mulheres na pós-menopausa com evidências de efeitos benéficos seletivos em outros orgãos. Outros beneficios potenciais de raloxifeno como proteção cardiovascular e prevenção de câncer de mama estão sendo investigados em grandes estudos clínicos a longo prazo. (Arq Bras Endocrinol Metab 1999; 43/6: 433-441)

Unitermos: Raloxifeno; Estrógeno; SERM; Osteoporose; Câncer de mama; Lipides

\footnotetext{
ABSTRACT

Raloxifene is a selective estrogen receptor modulator of second generation with agonist effect in the bone, cardiovascular system, and antagonist effect in the breast and uterus. The tissue selectivity of raloxifene occurs due to several mechanisms such as different estrogen receptors, differential distribution of receptors, different protein transcriptional factors and receptor conformation after raloxifene binding. In bone, raloxifene increases the bone mass in the spine, femur and total body, prevents osteoporosis in postmenopausal women and reduces the incidence of vertebral fractures in $50 \%$ in women with osteoporosis. In the cardiovascular system, raloxifene decreases total cholesterol, LDL-cholesterol, fibrinogen and lipoprotein (a), without changes in triglycerides and $\mathrm{HDL}$-cholesterol, however, it increases the subfraction $\mathrm{HDL}-\mathrm{C}_{2}$. Raloxifene has antiproliferative activity in the breast, does not induce mastalgia and a reduction in the incidence of new cases of breast cancer has been found in women taking raloxifene in the large osteoporosis trials. In the uterus, raloxifene does not stimulate the endometrium and does not increase the incidence of vaginal bleeding or endometrial car-
}

\author{
Disciplina de Endocrinologia da \\ Escola Paulista de Medicina - \\ Universidade Federal de São Paulo \\ (UNIFESP/EPM), SP e Eli Lilly do \\ Brasil Ltda.
}


cinoma. The most common adverse event with raloxifene is hot flashes and the most serious is venous thromboembolism with similar incidence as hormonal replacement therapy. Raloxifene is an alternative with evidence of selective beneficial effects in other tissues. Other potential benefits with raloxifene such as cardiovascular protection and breast cancer prevention are being investigated in long-term clinical trials. (Arq Bras Endocrinol Metab 1999; 43/6: 433-441)

Keywords: Raloxifene; Estrogen; SERM; Osteoporosis; Breast cancer; Lipids

$\mathrm{C}$ LORIDRATO DE RALOXIFENO É UM DERIVADO benzotiofênico, originalmente investigado para tratamento de câncer de mama avançado e descoberto há quase 20 anos (1). Este composto está classificado como um modulador seletivo do receptor de estrógeno (SERM) de segunda geração baseado em seu efeito tecido-específico nos orgãos alvo de ação do estrógeno. O termo SERM tem sido recentemente usado para descrever compostos que interagem com o receptor de estrógeno, porém possuem atividades tecido-específicas (2). Esta ação tecidoseletiva foi primeiramente descrita com tamoxifeno, um SERM de primeira geraçăo, que possui uma ação antiestrógena na mama, efeitos benéficos na massa óssea de mulheres pós-menopausadas, reduz o colesterol, porém estimula a proliferação endometrial (3-7). Entretanto, o uso clínico para tratar osteoporose é limitado devido a seu perfil de eventos adversos que inclui indução de hiperplasia e carcinoma endometrial, tromboflebite, alteração de função hepática e tumores hepáticos cm roedores (3). Os antagonistas estrogênicos completos (ICI-164.384 ou ICI-182.780), por outro lado, não têm habilidade de mimetizar os efeitos estrogênicos nos orgãos alvos, e produzem efeitos antagonistas tanto em tecidos reprodutores como no osso e provavelmente lípides, o que limita o seu uso clínico (4).

Como tamoxifeno, o raloxifeno possui ação antireabsortiva no osso, reduz o colesterol e possui ação antiproliferativa na mama. Porém, raloxifeno não causa estimulação endometrial (tabela I). Em estudos clínicos recentes, raloxifeno mostrou efeitos benéficos no esqueleto $(8,9)$, o que levou à aprovação do ralo- xifeno para a prevenção e tratamento de osteoporose no Brasil. Nesta revisão, abordaremos aspectos préclínicos/clínicos gerais atuais do raloxifeno e suas perspectivas para o futuro.

\section{ESTRUTURA QUÍMICA}

O termo anti-estrógeno foi originalmente aplicado a uma série de compostos que possuíam a habilidade de antagonizar os efeitos estimulatórios e proliferativos do estrógeno (7). Estes compostos têm alta afinidade pelo receptor de estrógeno $\mathrm{c}$ induzem respostas mediadas por estes receptores em tecidos reprodutores e não reprodutores. Várias classes químicas estão representadas neste grupo de compostos, incluíndo derivados trifeniletilenos (tamoxifeno, clomifeno). $\mathrm{O}$ raloxifeno representa um derivado benzotiofênico. $\mathrm{O}$ clomifeno já está em uso clínico há vários anos para tratamento de infertilidade feminina, enquanto droloxifeno, idoxifeno e toremifeno ainda estão em fase II/III de pesquisas clínicas. A estrutura do raloxifeno está representada na figura 1. A parte inferior da estrutura do raloxifeno liga-se ao receptor de estrógeno, enquanto a cadeia lateral básica se liga à um sítio diferente e leva às propriedades antagonistas do composto $(8,9)$.

\section{MECANISMO DE AÇĀO}

Os mecanismos pelos quais os SERMS têm efeitos agonistas/antagonistas de forma seletiva nos tecidos ainda não são completamente conhecidos. Raloxifeno exerce atividade agonista no osso c sistema cardiovascular e é um antagonista na mama e útero. O raloxifeno possui afinidade pelo receptor de estrógeno semelhante ao $17 \beta$ estradiol $(18,25)$. Múltiplos receptores de estrógeno e vias pós-receptores com diferentes cofatores protéicos serian responsáveis por pelo menos parte da atividade seletiva (9-18) (tabela 2). O receptor de estrógeno pertence a uma grande família de receptores nucleares que inclui cortisol, testosterona, progesterona, hormônio tiroideano, cortisol e ácido retinóico. Dois subtipos de receptores de cstrógeno, ER $\alpha$ e ER $\beta$, foram identifica-

Tabela 1. Classificação e perfil de acão de alguns compostos ativos no receptor de estrógeno nos diversos tecidos.

\section{SERMs}

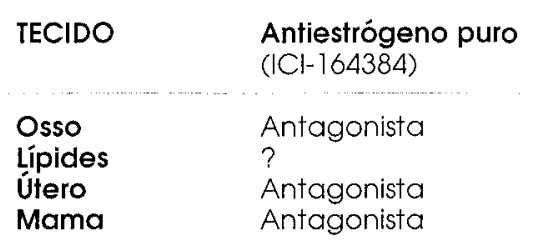

Primeira Geração
(Tamoxifeno)
Agonista
Agonista
Agonista Parcial
Antagonista

Segunda Geração
(Raloxifeno)
Agonista
Agonista
Antagonista
Antagonista

\section{Estrógenos} (17/-estradiol) 
<smiles>C[C@]12CCC3c4ccc(O)cc4CCC3C1CC[C@@H]2O</smiles>

17\%-estradiol

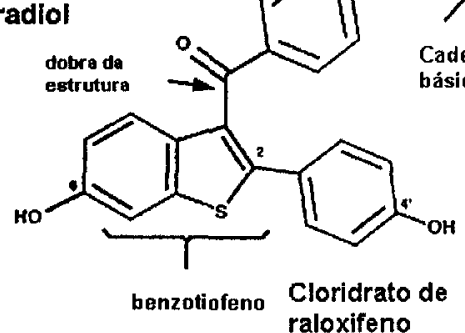

Figura1. Estrutura química do 17ß-estradiol e raloxifeno. A parte inferior da estrutura do raloxifeno se liga ao receptor de estrógeno, enquanto a cadeia lateral básica se liga à um sitio diferente e leva às propriedades antagonistas do composto $(8,9)$.

dos $(19,20)$, que podem interagir com elementos de resposta nos genes alvos promotores como homodímeros ou $\alpha \beta$ heterodímeros. Estes receptores exibem expressão diferencial nos diversos tecidos. Por exemplo, o RNA mensageiro (RNAm) do ER $\alpha$ têm alta expressão na hipófise, útero, testículo, rins e glândula adrenal $(19,20)$, enquanto os receptores beta são encontrados no ovário, testículo, próstata, timo e baço (2l-23). O RNAm do ER $\alpha$ e ER $\beta$ estão presentes nos osteoblastos, células epiteliais mamárias e no cérebro. Curiosamente, o receptor alfa predomina nos orgãos reprodutores (mama e útero), enquanto que o receptor beta predomina no osso e sistema cardiovascular $(21,23)$. Alguns estudos sugerem que raloxifeno é capaz de estimular as vias estrogênicas através do receptor beta, mas não é capaz de ativar via receptor alfa, podendo este ser un dos mecanismos pelos quais este fármaco exerce ação diferencial nos tecidos $(18,25)$.

O receptor de estrógeno possui múltiplas funções ativadoras transcricionais (sítios AF-l e AF-2) que contribuem para alguns dos efeitos seletivos do raloxifeno (24) Também, diferentes conformações do receptor induzidas pelos ligantes podem ser responsáveis pelo amplo efeito farmacológico dos vários SERMs (18). Como resultado de uma conformação própria receptor:raloxifeno, este complexo liga-se à sequências do DNA distintas no elemento de resposta estrogênica (ERE) em tecidos onde raloxifeno exerce efeitos agonistas ao estrógeno. Baseado na estrutura cristalográfica dos complexos formados por raloxifeno e $17 \beta$-estradiol com o domínio de ligação do receptor, evidenciou-se que
Tabela 2. Principais mecanismos pelos quais os Moduladores Seletivos do Receptor de Estrógeno (SERMS) possuem efeitos tecido-seletivos.

Alterações diferenciais da conformação do receptor após ligação do composto

Diferentes afinidades de ligação ao receptor de estrógeno

Ativação diferencial dos domínios de ativação do receptor de estrógeno

Interação do receptor de estrógeno com diferentes elementos de resposta no DNA

Interação com diferentes co-ativadores e corepressores proteicos nos tecidos

Interação dos ligantes com diferentes subtipos do receptor de estrógeno (ER $\alpha$ e ERR)

Expressão diferencial dos subtipos de receptores de estrógeno nos tecidos

os 2 compostos ligam no mesmo sítio; porém, exibem diferentes modos de ligação e induzem alterações conformacionais distintas no domínio de transativação (25), evidenciando uma base molecular para diferentes ações. Como exemplo, raloxifeno ativa o gene TGFß3 por um mecanismo de atividade transcricional de um gene que não contêm ERE (26). TGF $\beta$ é uma proteína abundante da matriz óssea com propriedades anti-osteoclásticas, cuja expressão in vitro é induzida por SERMs, mas não por $17 \beta$-estradiol (26). Este dado sugere a presença de um elemento de resposta de raloxifeno (ERR) no gene de TGF $\beta 3$ que é ativado via receptor de estrógeno por raloxifeno e de forma interessante não pelo estrógeno in vitro. Porém, in vivo tanto raloxifeno como $17 \beta$-estradiol produzem aumentos equivalentes dose-dependentes do RNAm de TGFB3 em fêmures de ratas ooforectomizadas (26). Possivelmente metabólitos do estradiol podem estar envolvidos na ativação do ERR ou ERE in vivo. Ativação de TGFß no tecido vascular pode ser também importante para os efeitos cardiovasculares dos SERMs também. Em ratos em dieta hipercolesterolêmica, tamoxifeno reduziu a lesão ateroesclerótica, tendo também elevado os níveis aórticos de TGFß (27).

\section{EFEITOS DO RALOXIFENO NOS DIVERSOS TECIDOS (TABELA 3)}

\section{Osso}

Os primeiros resultados com raloxifeno no tecido ósseo foram evidenciados em um modelo animal de deficiência estrogênica $\mathrm{cm}$ ratas ooforectomizadas. 
Após ooforectomia, há perda de osso trabecular evidente em 4 a 6 semanas, com mudanças em várias citoquinas, fatores de crescimento, e marcadores de remodelação óssea. Raloxifeno reduziu os níveis dos marcadores bioquímicos de metabolismo ósseo, restaurou os níveis de RNAm de TGF $\beta$ e reduziu os níveis de interleucina- 6 para níveis semelhantes às ratas não ooforectomizadas (controles) $(26,28)$. Neste modelo experimental, raloxifeno se mostrou tão eficiente quanto o estrógeno em preservar a densidade mineral óssea na coluna lombar, femur distal, e tíbia proximal. Estudos histomorfométricos no osso confirmaram que raloxifeno previne reabsorção do osso trabecular após ooforectomia de maneira similar ao estrógeno $(29,30)$.

\section{ESTUDOS DE PREVENÇĀO DE OSTEOPOROSE}

Os efeitos de raloxifeno nos marcadores bioquímicos de reabsorção óssea em mulheres na pós-menopausa evidenciam uma redução nos marcadores da formação óssea como osteocalcina e fosfatase alcalina ósseo específica, e marcadores da reabsorção óssea como a razão telopeptídeo do colágeno tipo $\mathrm{I} /$ creatinina urinária para níveis da pré-menopausa $(8,31)$. Em estudos de cinética de remodelação óssea, um balanço de cálcio positivo e redução da excreção urinária de

Tabela 3. Efeitos gerais do raloxifeno nos diversos tecidos.

Osso ( $8,9,26,28-33$ )
Previne perda óssea associada com deficiência estrogênica
Reduz o risco de fraturas vertebrais em mulheres osteoporóticas
Reduz a remodelação óssea em mulheres na pós-
menopausa (formaçáo e reabsorção óssea)
Diminui a excreção urinária de cálcio
Lípides ( $8,9,37,34)$
Diminui o colesterol total, LDL-colesterol e fibrinogênio
Não têm efeito nos triglicérides ou HDL-colesterol total
(aumenta a fração C 2 do HDL-colesterol)
Útero ( $8,9,33,51,52,54)$
Não estimula o endométrio ou miométrio
Inibe proliferação de células leiomiomatosas in vitro
Mama (42-50)
Redução do risco de câncer de mama
Sem efeito no tratamento de câncer de mama resistente
ao tamoxifeno
Inibe proliferação e invasividade de células cancerosas
mamárias
Inibe crescimento de tumores mamários em animais
Sistema Nervoso Central ( $8,9,31,57$ )
Sem efeito aparente na função cognitiva
Aumenta o crescimento dendritico neuronal de células PC-12
*Os números entre parênteses representam as referências
respectivas

Osso $(8,9,26,28-33)$

Previne perda óssea associ menopausa (formação e reabsorção óssea)

Diminui a excreção urinária de cálcio

Não têm efeito nos triglicérides ou HDL-colesterol total (aumenta a fração $\mathrm{C}_{2}$ do $\mathrm{HDL}$-colesterol)

Útero $(8,9,33,51,52,54)$

Não estimula o endométrio ou miométrio

Inibe proliferação de células leiomiomatosas in vitro

Redução do risco de câncer de mama

ao tamoxifeno

Inibe proliferação e invasividade de células cancerosas mamárias

Inibe crescimento de tumores mamários em animais

Sistema Nervoso Central $(8,9,31,57)$

Sem efeito aparente na função cognitiva

*Os números entre parênteses representam as referências espectivas cálcio foram observados (32). Em um estudo de prevenção em mulheres com densidade mineral óssea (DMO) normal, ou consideradas osteopênicas (definidas pelo critério da Organização Mundial da Saúde), raloxifeno, em doses variando de $30 \mathrm{mg}$ a 150 $\mathrm{mg}$, associado a $400 \mathrm{mg}$ a $600 \mathrm{mg}$ de cálcio elementar, reduziu os níveis dos marcadores bioquímicos de reabsorção óssea e aumentou significantemente a massa óssea (8). Aos 6 meses de estudo, já houve um incremento da massa óssea que se manteve no transcorrer dos 24 meses do estudo. Aos 2 anos de estudo, raloxifeno $(60 \mathrm{mg} /$ dia $)$ aumentou a $D M O$ na coluna e quadril total em $1,6 \%$, e no colo femural em $1,2 \%$. O conteúdo mineral ósseo total aumentou em $1,4 \%$. No grupo placebo cálcio-suplementado houve redução da DMO nas várias localidades em $0,6 \%$ a $1,3 \%$. Portanto, a diferença média na mudança da DMO comparando as mulheres que usaram raloxifeno com aquelas que receberam placebo foi de $2,4 \%$ na coluna lombar e quadril total, e $2,0 \%$ no corpo inteiro. A dose de $30 \mathrm{mg} /$ dia de raloxifeno mostrou-se menos eficaz que as doses de $60 \mathrm{mg} /$ dia ou $150 \mathrm{mg} /$ dia, não havendo diferença no incremento da DMO comparando-se as doses de $60 \mathrm{mg} /$ dia e $150 \mathrm{mg} /$ dia.

\section{ESTUDOS DE TRATAMENTO DE OSTEOPOROSE - IMPACTO SOBRE FRATURAS}

Em um estudo prospectivo, em 143 mulheres osteoporóticas pós-menopausadas com uma média de 68 anos de idade, com pelo menos uma fratura vertebral prevalente, comparou-se os efeitos de raloxifeno em doses variadas com um grupo placebo cálcio e vitamina D suplementado (9). Após 12 meses, raloxifeno na dose de $60 \mathrm{mg} /$ dia foi eficaz $\mathrm{cm}$ aumentar significantemente a DMO no quadril total em aproximadamente $1,7 \%$ e rádio ultradistal em $3,0 \%$. Houve também um aumento não significante de $1,8 \%$ na coluna lombar. Quando se usou um limite de corte de definição das vértebras de $30 \%$, houve uma redução significante da incidência de fraturas vertebrais com raloxifeno. Não houve diferença entre os grupos em relação às fraturas extra-vertebrais.

A eficácia de raloxifeno em prevenir fraturas em mulheres pós-menopausadas com osteoporose também está sendo avaliada em outro estudo multicêntrico global (estudo MORE). Este estudo placebo-controlado selecionou 7.705 mulheres (média de idade, 66,5 anos) osteoporóticas com ou sem fraturas vertebrais no início do estudo. O estudo têm previsão de duração de 4 anos, e está atualmente em seu último ano de duraçăo. Todas as mulheres foram designadas aleatoriamente para receber 
raloxifeno $60 \mathrm{mg} / \mathrm{dia}$ ou $120 \mathrm{mg} /$ dia e foram suplementadas com cálcio $(500 \mathrm{mg} /$ dia de cálcio elementar) e vitamina D (400-600 UI/dia). Resultados intermediários de 3 anos (33) evidenciaram uma redução significante na incidência de novas fraturas vertebrais de $30 \%$ a $50 \%$ tanto nas mulheres sem fraturas como naquelas com fraturas vertebrais no início do estudo, respectivamente. A DMO aumentou significantemente de $2 \%$ a $3 \%$ no quadril, coluna e corpo inteiro em 24 meses. Este estudo não tem poder estatístico suficiente para detectar redução no risco de fraturas extra-vertebrais, já que o número de fraturas extra-vertebrais incidentes neste estudo foi baixa.

Estudos que comparam diretamente o efeito de raloxifeno com estrógeno ou alendronato não foram publicados, não permitindo uma avaliação comparativa precisa. Entretanto, o ganho absoluto de DMO no quadril parece ser semelhante nas 3 terapêuticas, enquanto apesar de ter um impacto semelhante na redução de fraturas vertebrais que outras terapêuticas anti-reabsortivas, o ganho de DMO absoluto na coluna lombar parece ser menor com raloxifeno.

\section{HISTOMORFOMETRIA ÓSSEA}

Em um estudo cego com 59 mulheres na pósmenopausa comparou-se o efeito de raloxifeno nas doses de $60 \mathrm{mg} /$ dia ou $120 \mathrm{mg} /$ dia comparado ao placebo. A análise histomorfométrica de amostras obtidas através de biópsias transilíacas após 6 meses, não evidenciou osteomalácia, lesão de osteócito, tecido ósseo desorganizado, fibrose medular ou outras anormalidades (dados de arquivo, Lilly).

\section{LÍPIDES}

Os efeitos do raloxifeno sobre parâmetros de risco cardiovascular foram avaliados em vários estudos de prevenção e tratamento para osteoporose $(8,9,31$, 34). Nestes estudos, observa-se uma redução do colesterol total (em média de $8 \%$ ), redução do LDLcolesterol $(-11 \% \mathrm{em}$ relação ao início do estudo), redução dos níveis de fibrinogênio $(-12 \%$ a $14 \%)$ e Lipoproteína (a) $(-7 \%$ a $8 \%)$ e nenhum efeito sobre o HDL-colesterol e triglicérides. Dados epidemiológicos sugerem que níveis elevados de fibrinogênio e lipoproteína (a) podem isoladamente constituir fatores preditivos quanto ao advento de doença cardiovascular $(35,36)$.

Um estudo de 6 meses em 390 mulheres pósmenopausadas comparou o efeito de doses variadas de raloxifeno a da terapia de reposição hormonal (TRH) contínua combinada $(0,625 \mathrm{mg}$ de estrógenos conju- gados equinos mais $2,5 \mathrm{mg}$ de acetato de medroxiprogesterona) e ao placebo (34). TRH e raloxifeno em todas as doses foram semelhantes em reduzir o colesterol total e LDL-colesterol. Raloxifeno não teve efeito em aumentar o HDL-colesterol total como TRH, porém, aumentou significantemente o nível da subfração do colesterol $\mathrm{HDL}-\mathrm{C}_{2}(15 \%$ em relação ao basal). Raloxifeno também não afetou os níveis de triglicérides, enquanto TRH aumentou os triglicérides em 20\%. Raloxifeno reduziu significantemente os níveis séricos de fibrinogênio em $12 \%$ a $14 \%$, enquanto TRH não teve efeito significante neste parâmetro.

Em adição ao seu efeito hipocolesterolêmico, raloxifeno pode ter outros efeitos cardioprotetores devido a propriedades antioxidantes. Raloxifeno inibc a oxidação do LDL em macrófagos peritoneais de camundongos (37). Em coelhos, raloxifeno inibe o acúmulo de colesterol aórtico (38). Porém, em um estudo em macacos raloxifeno não teve efeitos protetores quanto à prevenção de ateroesclerose coronariana, apesar de ter reduzido as concentrações de colesterol (39). Porém, neste mesmo estudo, doses habituais de reposição estrogênica também não foram eficazes em inibir a ateroesclerose, necessitando de doses supra-fisiológicas para se ter este efeito (40). A diferença entre os dois estudos pode ser devido à espécies animais diferentes, modelos de ateroesclerose diversos, doses das drogas, duração da terapêutica, dieta, ou métodos usados para quantificação da extensão da ateroesclerose (4l). Não há dados relativos ao efeito de raloxifeno sobre a vasoconstriç̧ão arterial ou interferência no metabolismo de glicose ou insulina, efeitos que são frequentemente relacionados à $\mathrm{TRH}$. Resultados à longo prazo em relação à proteção para doenças cardiovasculares são necessários em humanos.

\section{MAMA}

Raloxifeno apresenta atividade inibitória estrogênica no tecido mamário in vitro. Em estudos pré-clínicos, raloxifeno mostrou-se mais potente que tamoxifeno em inibir proliferação induzida pelo estrógeno de células tumorais mamárias humanas e em outras linhagens celulares $(42,43)$. Raloxifeno inibe também o desenvolvimento de tumores mamários em ratas in vivo, incluindo aqueles induzidos pela nitrosometiluréia ou 7,12-dimetilbezantraceno (DMBA) (43-46). Tumores estrógenoindependentes não são inibidos por raloxifeno. Em pacientes com câncer de mama disseminado resistentes ao tamoxifeno, raloxifeno também não produziu resposta de remissão parcial ou completa no tumor (47). 
A incidência de câncer de mama tem sido avalıada nos estudos clínicos com raloxifeno para prevenção e tratamento de osteoporose $(48,49)$. Em estudo publicado recentemente com um número grande de pacientes, o uso de raloxifeno reduziu em $76 \%$ a incidência de novos casos de câncer de mama invasivos, após uma média de 40 meses de acompanhamento em mulheres na pós-menopausa (50). Neste mesmo estudo, após classificação dos tumores conforme seu status, receptor de estrógeno negativo ou positivo (ER- ou ER+), raloxifeno reduziu em 90\% a incidência de novos tumores ER+ diagnosticados, não tendo efeito na incidência de tumores ER-. Portanto, raloxifeno pode se mostrar uma alternativa viável ao tratamento de reposição hormonal para prevenção e tratamento de osteoporose em mulheres com história pessoal ou familiar de câncer de mama e mulheres que não queiram usar estrógeno devido à seus efeitos colaterais ou medo de câncer.

\section{ÚTERO}

Comparado ao estrógeno ou tamoxifeno, raloxifeno não exerce atividade agonista no tecido uterino (51). Em animais, raloxifeno age com um antagonista no endométrio. Nestes estudos pré-clínicos, a hipertrofia endometrial induzida por estradiol e o crescimento de células de carcinoma endometrial estimulado por tamoxifeno são inibidos por raloxifeno $(25,51)$. Resultados de estudos clínicos em mulheres na pósmenopausa também não demonstram efeitos estimulatórios no útero $(8,9,33,52)$. Nestes estudos, não há mudança na espessura endometrial ou desenvolvimento de endométrio proliferativo durante terapêutica com raloxifeno e não se detectou aumento na incidência de sangramento vaginal ou de carcinoma endometrial $(33,52)$. Em mulheres na pré-menopausa, não foram detectados efeitos antagonistas significativos à nível de endométrio, não havendo qualquer mudança do ciclo menstrual, alteração na ovulação, secreção de gonadotrofinas, estradiol, e secreção de progesterona durante o ciclo menstrual (53). Portanto, parece que no estado de deficiência estrogênica (pós-menopausa), raloxifeno age como um antagonista estrogênico e não estimula o útero. Em mulheres com altos níveis de estrógeno (pré-menopausa) há atenuação deste efeito antagonista no útero. Apesar de efeitos benéficos in vitro e em modelos animais, é improvável que raloxifeno seja útil no tratamento de doenças responsivas ao estrógeno, como endometriose ou leiomioma uterino. Vários análogos de raloxifeno estão em investigação para aplicações potenciais nestas doenças (54).

\section{MISCELÂNEA}

Não há alteração ponderal ou de sinais vitais em uso de raloxifeno $(8,9,31)$. Em estudos para osteoporose, não se observou qualquer efeito na função cognitiva associada ao uso de raloxifeno $(8,9,31,55)$. Estudos da função cognitiva a longo prazo estão em andamento. Os efeitos de raloxifeno na doença de Alzheimer também são desconhecidos. Para avaliar o efeito de raloxifeno sobre o eixo hipotalâmico-hipofisário-gonadal, os níveis séricos de FSH e LH de mulheres na pósmenopausa foram medidos em um cstudo de 2 meses, que incluía altas doses de raloxifeno $(200 \mathrm{mg} / \mathrm{dia} e$ $600 \mathrm{mg} /$ dia), e em um estudo de 3 anos de prevenção de osteoporose em um subgrupo de indivíduos. No estudo a curto prazo, as alterações nos níveis séricos de FSH e LH nas mulheres tratadas com raloxifeno não foram estatisticamente significantes (dados de arquivo, Lilly). No estudo a longo prazo, os níveis séricos de FSH foram significantemente reduzidos, com uma redução média de $23 \%$ no grupo do raloxifeno ( 60 $\mathrm{mg} /$ dia) comparado a uma redução média de $8 \%$ no grupo placebo (dados de arquivo, Lilly). No mesmo estudo, indivíduos tomando estrógenos equinos conjugados $(0,625 \mathrm{mg} / \mathrm{dia})$ tiveram uma redução média de $40 \%$ nos níveis séricos de FSH. Neste estudo, os efeitos sobre o FSH de ambos os tratamentos ativos foram significantemente diferentes do placebo, e o efeito do raloxifeno foi significantemente diferente do efeito dos estrógenos equinos conjugados. Na avaliação final, não houve diferenças sobre os níveis de LH com placebo, raloxifeno, ou estrógenos equinos conjugados (dados de arquivo, Lilly). O efeito do raloxifeno sobre o FSH sérico pareceu representar uma ação estrogênica do raloxifeno.

\section{EVENTOS ADVERSOS}

A incidência de ondas de calor em mulheres em uso de raloxifeno tem sido indicada como superior à das mulheres em uso de estrógenos, porém não diferente de placebo em alguns estudos $(8,9,31)$. Porém, em outros estudos, raloxifeno foi associado a um aumento de até 7\% na incidência de ondas de calor em relação ao placebo, geralmente nos primeiros 6 meses de tratamento (dados de arquivo, Lilly). Porém, o índice de descontinuação por este sintoma não é maior do que nas mulheres em uso de placebo. $O$ índice de sangramento vaginal e mastalgia nas mulheres em uso de raloxifeno é semelhante ao do grupo placebo, entretanto é menor àquela referida com estrógenos $(8,9,34)$. Outro evento adverso observado com ralo- 
xifeno foram cãibras nos membros inferiores que não se relacionaram com qualquer distúrbio eletrolítico ou miopatia. O índice de descontinuação por este sintoma também não foi diferente em relação ao grupo placebo. O efeito colateral mais grave associado com raloxifeno foi um aumento em 2 a 3 vezes no risco de troboembolismo venoso, um aumento de risco similar à terapêutica estrogênica (dados de arquivo, Lilly). O risco estimado desta ocorrência seria de 2 a 3 casos por 10000 mulheres por ano (56).

\section{INDICACĀO}

Raloxifeno na dose de $60 \mathrm{mg} /$ dia está aprovado para o uso na prevenção e tratamento de osteoporose $\mathrm{cm}$ mulheres na pós-menopausa. Raloxifeno mostroumse eficaz em aumentar a DMO e reduzir a incidência de fraturas vertebrais em mulheres com osteoporose na pós-menopausa. Raloxifeno não deve ser utilizado em mulheres que apresentem sintomas agudos importantes da menopausa, como ondas de calor e ressecamento vaginal, e que necessitem ou desejem usar terapia de reposição hormonal para alívio destes sintomas. Não existem dados de segurança ou efiććcia quanto ao uso combinado de raloxifeno e terapia de reposição hormonal. Dados relativos ao uso de raloxifeno $\mathrm{cm}$ homens também não estão disponíveis, não se conhecendo portanto seu efeito nos homens.

\section{DESAFIOS FUTUROS/PERSPECTIVAS}

SERMs, como raloxifeno, que possuem um perfil de segurança melhorado no tecido reprodutor, representam uma alternativa potencial importante à terapia de reposição hormonal em mulheres na pós-menopausa para tratamento e prevenção de osteoporose e doença cardiovascular. Questões importantes ainda têm que ser investigadas. Apesar de raloxifeno demonstrar um impacto positivo nos lípides séricos, a possível proteção cardiovascular ainda deve ser estabelecida em estudos a longo prazo. Com esse objetivo, um grande estudo global multicêntrico (estudo RUTH) envolvendo 10.000 mulheres na pós-menopausa com risco de doença cardiovascular em 26 países foi iniciado em 1998. Estas mulheres serão estudadas por mais de 5 anos para avaliar o efeito de raloxifeno na prevenção primária e secundária de eventos e mortalidade cardiovasculares. Outro grande potencial para raloxifeno seria a prevenção de câncer de mama diante de dados de redução da incidência de novos casos de câncer de mama em mulheres nos estudos para osteoporose. Um grande estudo (estudo STAR) envolvendo 22.000 mulheres pós-menopausadas com risco elevado de câncer de mama está se iniciando em 1999 nos Estados Unidos, Canadá e Porto Rico para estudar o efeito de raloxifeno versus tamoxifeno na prevenção de câncer de mama e deverá ter a duração de 5 anos. Os resultados destes 2 grandes estudos serão importantes para que tenhamos evidências prospectivas dos beneficios do raloxifeno a longo prazo.

\section{REFERÊNCIAS}

1. Black LJ, Jones CD, Falcone JF. Antagonism of estrogen action with a new benzotiophene derived antiestrogen. Life Sci 1983:32:1031-6.

2. Mitlak $\mathrm{BH}$, Cohen FJ. In search of the optimal long-term female hormone replacement: the potential of selective receptor modulators. Horm Res 1997;48:155-63.

3. Davidson NE. Tamoxifen-Panacea or Pandora's box? N Engl J Med 1992;326:885-6.

4. van Leeuwen FE, Benraadt J, Coebergh JW, Kiemeney LA, Gimbrere CH. Otter R, et al. Risk of endometrial cancer after tamoxifen treatment of breast cancer. Lancet $1994 ; 343: 448-52$.

5. Love RR, Mazess RB, Barden HS, Epstein S, Newcomb PA, Jordan VC, et al. Effects of tamoxifen on bone mineral density in postmenopausal women with breast cancer. N Engl J Med 1992;326:852-6.

6. Grey AB, Stapleton JP. Evans MC. Tatnell MA, Ames RW. Reid IR. The effect of the antiestrogen tamoxifen on bone mineral density in normal late postmenopausal women. Am J Med 1995:99:636-41.

7. Bryant HU, Dodge JA, Sato M, Glasebrook AL. Comparative pharmacological profiles for a spectrum of estrogen receptor active agents in ovariectomized rats. Osteoporosis Int 1996:6:233-6.

8. Delmas PD, Bjarnason NH, Mitlak BH, Ravoux AC, Shah AS, Huster WJ, et al. Effects of raloxifene on bone mineral density, serum cholesterol concentrations, and uterine endometrium in postmenopausal women. N Engl J Med 1997:337:1641-7

9. Lufkin EG, Whitaker MD. Nickelsen T, Argueta R; Caplan $\mathrm{RH}$, Knickerbocker RK. Treatment of established postmenopausal osteoporosis with raloxifene: a randomized trial. J Bone Miner Res 1998; 13:1747-54

10. Lerner LJ, Jordan VC. Development of antiestrogens and their use in breast cancer: eighth Cain Memorial Award Lecture. Cancer Res 1990:50:4177-89.

11. Grese TA, Cho S, Finley DR, Godfrey AG, Jones $C D$, Lugar CW, et al. Structure-activity relationships of selective estrogen receptor modulatros: modifications to the 2-arylbenzothiophene core of raloxifene. J Med Chem. 1997;40: 146-67.

12. Yang NN, Venugopalan M, Hardikar S, Glasebrook $A$ Identification of an estrogen response element activated by metabolites of $17 \mathrm{~b}$-estradiol and raloxifene. Science 1996:273:1222-5.

13. Cheskis BJ, Karathanasis S, Lyttle CR. Estrogen receptor ligands modulate its interaction with DNA. J Biol Chem 
1997:272:11384-91.

14. Halachmi S, Marden E, Martin G, Mackay H, Abbondanza C. Brown M. Estrogen receptor-associated proteins: possible mediators of hormone-induced transcription. Science 1994:264:1455-8.

15. Weiss DJ, Gurpide E. Non-genomic effects of estrogens and anti-estrogens. Breast Cancer Res Treat 1994;3 1:5-9.

16. Couse JF, Lindzey J, Grandien K, Gustafsson JA, Korach KS. Tissue distribution and quantitative analysis of estrogen receptor-alpha (Eralpha) and estrogen receptorbeta (Erbeta) messenger ribonucleic acid in the wildtype and Eralpha-knockout mouse. Endocrinol 1997: 138:4613-21.

17. Greese TA, Sluka JP, Bryznat HU, Cullinan GJ, Glasebrook $\mathrm{AL}$, Jones $\mathrm{CD}$, et al. Molecular determinants of tissue selectivity in estrogen receptor modulators. Proc Natl Acad Sci USA. 1997;94:14105-10.

18. McDonnell DP, Clemm DL, Hermann T, Goldman ME, Pike JW. Analysis of estrogen receptor function in vitro reveals three distinct classes of antiestrogens. Mol Endocrinol 1995:9:659-69.

19. Kuiper GG, Enmark E, Pelto-Huikko M, Nilsson S, Gustafsson JA. Cloning of a novel estrogen receptor expressed in rat prostate and ovary. Proc Natl Acad Sci USA. 1996:93:5925-30.

20. Mosselman S, Ploman J, Dijkema R. Erb: identification and characterization of a novel human estrogen receptor. FEBS Lett. 1996;392:49-53.

21. Arts J, Kuiper GG, Janssen JM, Gustafsson JA, Lowik CW. Pols HA, et al. Differential expression of estrogen receptors alpha and beta mRNA during differentiation of human osteoblast SV-HFO cells. Endocrinology 1997; 138:5067-70.

22. Dotzlaw H. Leygue E, Watson PH, Murphy LC. Expression of estrogen receptor-beta in human breast tumors. J Clin Endocrinol Metab 1996;82:2371-4.

23. Shughrue PJ, Lane MV, Merchenthaler I. Comparative distribution of estrogen receptor-alpha and-beta mRNA in the rat central nervous system. J Comp Neurol 1997:388:507-25.

24. Bryant HU, Dere WH. Selective estrogen receptor modu lators: an alternative to hormone replacement therapy. P.S.E.B.M. 1998;217:45-52.

25. Brzozowski AM, Plke AC, Dauter Z, Hubbard RE, Bonn T, Engstrom $O$, et al. Molecular basis of agonism and antagonism in the oestrogen receptor. Nature $1997: 389: 753-8$

26. Yang NN, Hardikar S, Sato M, Galvin RJS, Glasebrook AL, Bryant $\mathrm{HU}$, et al. Estrogen and raloxifene stimulate transforming growth factor-b $b_{3}$ expression in rat bone: a potential mechanism for estrogen- or raloxifene-mediated bone maintenance. Endocrinology 1996; 137:2075-84

27. Grainger DJ, Wittchell CM. Metcalfe JC. Tamoxifen elevates transforming-growth factor-beta and supresses diet-induced formation of lipid lesions in mouse aorta Nat Med 1995: 1:1067-73.

28. Glasebrook AL, Short LL, Cole HW, Magee DE, Bryant HU. Regulation of serum IL-6 by raloxifene in an ovariectomized rat model (Abstract). Bone 1995; 16:99S.
29. Evans $G$, Bryant HU, Magee D, Sato M, Turner RT. The effects of raloxifene on tibia histomorphometry in ovariectomized rats. Endocrinology 1994;134:2283-8.

30. Sato M. Bryant HU, Iversen P. Helterbrand J. Smietana F. Bemis $K$, ef al. Advantages of raloxifene over alendronate or estrogen on nonreproductive and cancer cells. Breast cancer Res Treat 1989: 14:65-76.

31. Draper MW, Flowers DE, Huster WJ, Neild JA, Harper KD. Amaud C. A controlled trial of raloxifene ( $L Y$ 139481) $\mathrm{HCl}$ : impact on bone turnover and serum lipid profile in healthy postmenopausal women. J Bone Miner Res $1996 ; 11: 835-42$

32. Heaney RP. Draper MW. Raloxifene and estrogen: comparative bone-remodeling kinetics. J Clin Endocrinol Metab 1997; 82:3425-9

33. Ettinger B, Black D, Mitlak BH, Knickerbocker RK, Nickelsen T, Genant H, et al. Reduction of vertebral fracture risk in posmenopausal women with osteoporosis treated with raloxifene - results from a 3-year randomized clinical trial. JAMA 1999:282:637-45.

34. Walsh BW, Kuller LH, Wild RA, Paul S, Farmer M, Lawrence $\mathrm{JB}$, et al. Effects of raloxifene on serum lipids and coagulation factors in healthy postmenopausal women. JAMA 1998; 279: 1445-51

35. Scanu AM. Lipoprotein (a). A genetic risk factor for premature coronary heart disease. JAMA 1992;267:3326-9.

36. Kannel WB, Wolf PA, Castelli WP, D'Agostino RB. Fibrinogen and risk of cardiovascular disease. The Framingham study. JAMA 1987;258:1183-6.

37. Zuckerman SH. Bryan N. Inhibition of LDL oxidation and myeloperoxidase dependent tyrosyl radical formation by the selective estrogen receptor modulator raloxifene (LY $139481 \mathrm{HCl}$ ). Atherosclerosis 1996; 126:65-75.

38. Bjarnason NH, Haarbo J, Byrjalsen I, Kauffman RF, Christiansen $\mathrm{C}$. Raloxifene inhibits aortic accumulation of cholesterol in ovariectomized cholesterol-fed rabbits. Circulation 1997; 96:1964-9.

39. Clarkson TB, Anthony MS. Jerome CP. Lack of effect of raloxifene on coronary artery atherosclerosis of postmenopausal monkeys. J Clin Endocrinol Metab $1998: 83: 721-6$.

40. Bryant HU, Kauffman RF, Iversen P, Cox DA, Mitlak BH, Heath III $\mathrm{H}$. Comment on lack of effect of raloxifene on coronary artery atherosclerosis of postmenopausal monkeys. J Clin Endocrinol Metab 1998;83:3001-2.

41. Khovidhunkit W., Shoback DM. Clinical effects of raloxifene hydrochloride in women. Ann Intern Med 1999; 130:437-9

42. Balfour JA, Goa KL. Raloxifene. Drugs and Aging $1998 ; 12: 335-41$

43. Wakeling AE, Valcaccia B, Newboult E, Green LR. Nonsteroidal antioestrogens-receptor binding and biological response in rat uterus, rat mammary carcinoma and human breast cancer cells. J Steroid Biochem $1984 ; 20: 117-20$

44. Clemens JA, Bennett DR, Black LJ, Jones CD. Effects of a new estrogen, keoxifene (LY 156758), on growth of carcinogen-induced mammary tumors and on LH and prolactin levels. Life Sci 1983;32:2869-75. 
45. Anzano MA, Peer CW, Smith JM, Mullen LT, Shrader MW Logsdon, et al. Chemoprevention of mammary carcinogenesis in the rat: combined use of raloxifene and 9-cisretinoic acid. J Nall Cancer Inst 1996:88:123-5.

46. Gottardis MM, Jordan VC. Antitumor actions of keox ifene and tamoxifen in the $\mathrm{N}$-nitrosomethylurea-induced rat mammary carcinoma model. Cancer Res $1987 ; 47: 4020-4$

47. Buzdar AU, Marcus C, Holmes F, Hug V. Hortobagyi $G$. Phase II evaluation of LY 156758 in metastatic breast cancer. J Cell Biochem Suppl 1995:22:51-7.

48. Cummings SR, Norton L, Eckert S, Grady D, Cauley J. Knickerbocker $R$, et al. Raloxifene reduces the risk of breast cancer and may decrease the risk of endometrial cancer in post-menopausal women. Two-year findings from the multiple outcomes of raloxifene evaluation (MORE) trial (Abstract). Proceedings of the American Society of Clinical Oncology Meeting 1998:3.

49. Jordan VC, Glusman JE, Eckert S, Lippman M, Powles T, Costa $T$, et al. Incident primary breast cancers are reduced by raloxifene: integrated data from multicenter, double-blind, randomized trials in 12,000 postmenopausal women (Abstract). Proceedings of the American Society of Clinical Oncology Meeting 1998:466.

50. Cummings SR, Eckert S, Krueger KA, Grady D, Powles TJ Cauley JA, et al. The effect of raloxifene on risk of breast cancer in postmenopausal women - results from the MORE randomized trial. JAMA 1999:281:2189.

51. Gottardis MM, Ricchio ME, Satyaswaroop PG, Jordan VC. Effect of steroidal and nonsteroidal antiestrogens on the growth of a tamoxifen-stimulated human endometrial carcinoma (EnCa 101) in athymic mice. Cancer Res 1990:50:3189-92.
52. Boss SM, Huster WJ, Neild JA, Glant MD, Eisenhut CC, Draper MW. Effects of raloxifene hydrochloride on the endometrium of postmenopausal women. Am J Obstet Gynecol 1997; 177:1458-64.

53. Baker VL, Draper $M$, Paul S, Allerheiligen S, Glant $M$, Shifren J, et al. Reproductive endocrine and endometrial effects of raloxifene hydrochloride, a selective estrogen receptor modulator, in women with regular menstrual cycles. J Clin Endocrinol Metab 1998;883:6-13.

54. Fuchs-Young R, Howe S, Hale L, Miles R, Walker C. Inhibition of estrogen-stimulated growth of uterine leiomyomas by selective estrogen receptor modulators. Mol Carcinog 1996;17:151-9.

55. Nickelsen T, Lufkin EG, Riggs BL, Cox DA, Crook TH. Raloxifene hydrochloride, a selective estrogen receptor modulator: Safety assessment of effects on cognitive function and mood in postmenopausal women. Psychoneuroendocrinol 1999:24:115-28.

56. Delmas PD, Mitlak BH. Christiansen C. Effects of raloxifene in postmenopausal women (Letter). N Engl J Med 1998;338:1313-4.

57. Nilsen J, Mor G, Naftolin F. Raloxifene induces neurite outgrowth in estrogen receptor positive PC12 cells. Menopause 1998:5:211-6.

\section{Endereço para correspondência:}

Marcia J. Kayath

Eli Lilly do Brasil

Avenida Morumbi 8264

04703-002 - São Paulo, SP

e-mail: kayath_marcia_jeha@|illy.com 